\title{
Correspondence
}

We welcome letters to the Editor concerning articles which have recently been published. Such letters will be subject to the usual stages of selection and editing; where appropriate the authors of the original article will be offered the opportunity to reply.

Letters should normally be under 300 words in length, double-spaced throughout, signed by all authors and fully referenced. The edited version will be returned for approval before publication.

\section{Extracorporeal shock-wave therapy for tendonitis of the rotator cuff}

Sir,

I read with interest the article in the May 2002 issue by Speed et $\mathrm{al}^{1}$ entitled 'Extracorporeal shock-wave therapy (ESWT) for tendonitis of the rotator cuff'.

I agree with others ${ }^{2,3}$ that double-blind randomised controlled trials are needed for better understanding of the true therapeutic value of ESWT in specific musculoskeletal conditions. To reach this goal, however, specific conditions must be well defined by strict admission and exclusion criteria.

With regard to non-calcific tendonitis of the rotator cuff, the clinical signs and symptoms are so close to those of partial-thickness tears of the cuff that a clinical differential diagnosis between the two can be difficult and misleading. Cases of tear of the rotator cuff may have been misdiagnosed as tendonitis and admitted to the study. Moreover, the mean age of the admitted patients (over 50 years) is more in favour of tears than tendonitis. ${ }^{4}$ Tears may be differentiated from tendonitis by sonography or MRI, but these were not used in this study.

In a trial designed to study the supposed therapeutic effect of ESWT on tendonitis, the results require a very different interpretation if some cases studied are not due to tendonitis but to tears.

In the search for appropriate indications for ESWT, accurate definition of the specific pathological conditions is desirable. A possible overlap of the two conditions of tendonitis and tears of the rotator cuff is not acceptable.

F. ASTORE, MD

Universitá degli Studi di Milano

Milano, Italy.

1. Speed CA, Richards C, Nichols D, et al. Extracorporeal shock-wave therapy for tendonitis of the rotator cuff: a double-blind, randomised, controlled trial. J Bone Joint Surg [Br] 2002;84-B:509-12.

2. Ogden JA, Alvarez RG, Levitt R, Marlow M. Shock wave therapy (Orthotripsy®) in musculoskeletal disorders. Clin Orthop 2001;387: 22-40.

3. Rompe JD, Wirth CJ. Application of extracorporeal shock-wave therapy in orthopaedics: where do we stand? Orthopäde 2002;31:609.

4. Neer CS II. Anterior acromioplasty for the chronic impingement syndrome of the shoulder. J Bone Joint Surg [Am] 1972;54-A:41-50.

(C)2003 British Editorial Society of Bone and Joint Surgery

doi:10.1302/0301-620X.85B5.14626 \$2.00

J Bone Joint Surg [Br] 2003;85-B:774-7.

\section{Author's reply:}

Sir,

We thank Dr Astore for his comments relating to the diagnosis of tendinopathies of the rotator cuff. As we have stated, the inclusion criteria for our study were based upon clinical findings and in particular the absence of weakness suggestive of a considerable tear of the cuff. We agree that the differentiation between tendinosis and partial- and full-thickness tears is unclear, and that imaging provides a sensitive approach to discriminating between these complaints. We emphasise that the natural history and optimal management of all conditions are still not clear. We agree that the imaging of subjects in randomised, controlled trials of treatment strategies in tendinopathy of the rotator cuff may provide predictors of outcome to treatment.

C. A. SPEED

University of Cambridge

Cambridge, UK.

\section{Arthroplasty of the hip - leg length is not important}

Sir,

I read with interest the article in the April 2002 issue by White and Dougall ${ }^{1}$ entitled 'Arthroplasty of the hip - leg length is not important' and would welcome comments on two points.

First, the reader is not told whether the patients who had limblength discrepancy determined by orthoroentgenography actually noticed any inequality of leg length. In their series Edeen, Sharkey and Alexander ${ }^{2}$ reported that more than $50 \%$ of patients who noticed leg-length discrepancies were disturbed by their inequality.

Secondly, there is no mention of any fixed abduction or adduction deformities of the hip which may have been present before the arthroplasty and the outcome of any limb-length discrepancy on any fixed deformities. Shortening of the leg, either because of flexion deformity of the ipsilateral knee or shortening of the ipsilateral tibia or femur, are also not considered in the analysis.

S. SHARMA, FRCS Ed, FRCS G

Glasgow Royal Infirmary,

Glasgow, UK.

1. White TO, Dougall TW. Arthroplasty of the hip-leg length is not important. J Bone Joint Surg [Br] 2002;84-B:335-8.

2. Edeen J, Sharkey PF, Alexander AH. Clinical significance of leglength inequality after total hip arthroplasty. Am J Orthop 1995;24:34751.

\section{Authors' reply:}

Sir,

We thank Mr Sharma for his interest in our paper.

Our method of radiographic measurement of hip lengthening did not include assessment of the limb length. We did not look at flexion deformity in the opposite knee or shortening of the ipsilateral tibia or femur either clinically or radiographically. A hip replacement can only alter limb length at the hip, and while we agree that pre-existent deformities affect the results of arthroplasty, 
we wished to isolate the specific effects of hip lengthening in this study.

We did not specifically inform patients if they had had lengthening, since in so doing, a bias would be imported to the study. We did independently look at psychological function as part of the SF36 analysis and hip pain as a part of the Harris hip score and satisfaction with surgery. In our patients who had not been informed of limb lengthening, no relationship was found between these factors and lengthening. We would perhaps infer from our findings and from those of the study of Edeen et al $^{1}$ that the awareness of a limb-length inequality may result in patient disturbance rather than the variance in length itself.

Like all patients with arthritis of the hip many of our patients had adduction and fixed flexion deformities. These were measured and recorded by a research physiotherapist both before and after operation. We have not specifically looked at the effect of lengthening on these deformities as yet, since we felt that this additional information might detract from the main thesis of our paper.

\section{T. O. WHITE, AFRCS}

T. W. DOUGALL, FRCS Orth

Queen Margaret Hospital,

Dunfermline, UK

1. Edeen J, Sharkey PF, Alexander AH. Clinical significance of leg length inequality after total hip arthroplasty. Am J Orthop 1995;24:34751 .

\section{The role of angiography in the management of haemorrhage from major fractures of the pelvis}

Sir,

We read with interest the article by Cook et al ${ }^{1}$ in the March 2002 issue entitled 'The role of angiography in the management of haemorrhage from major fractures of the pelvis'. They obviously have extensive experience in the management of fractures of the pelvic ring. There seems no doubt that angiography and embolisation have an important role to play in a certain subset of these fractures.

The algorithm described in Figure 3 seems at odds with their discussion of the results. For patients with an increased pelvic volume, the discussion states that application of an external fixator should be the first procedure after fluid resuscitation. This is in accordance with ATLS protocols, which state that control of haemorrhage follows the resolution of airway and breathing problems. ${ }^{2}$ However, their algorithm suggests that diagnostic peritoneal lavage (DPL)/ultrasound (USS) should precede control of haemorrhage. Moreover, in their algorithm, the result of DPL/USS does not influence the next step, which is the application of a pelvic external fixator. Diagnostic investigations, such as DPL/USS, should be considered after control of haemorrhage as part of the secondary survey. We suggest that it would be more appropriate for their algorithm to read as follows. In hypotensive patients with an increased pelvic volume an external fixator should be applied. Following that, DPL/USS can be used to determine the need for laparotomy, a positive result being an indication for laparotomy. If DPL/USS is negative, angiography or CT may be performed.

M. MORAN, MRCS

R. SAMARJI, MCh, FRCS Orth

Manchester Royal Infirmary

Manchester, UK.

1. Cook RE, Keating JF, Gillespie I. The role of angiography in the management of haemorrhage from major fractures of the pelvis. J Bone Joint Surg [Br] 2002;84-B:178-82.

2. American College of Surgeons' Committee on Trauma. Advanced life support for doctors (student course manual). Sixth edition. 1997.
Author's reply:

Sir,

We thank Messrs Moran and Samarji for their comments. The algorithm in our article was not intended to be a comprehensive guide to the management of the hypotensive patient with multiple trauma. The main aim was to suggest an appropriate sequence of investigation leading to a logical decision to perform angiography in a selected group of patients. In particular, the algorithm was not intended to imply that no fluid resuscitation would be given before a diagnostic peritoneal lavage or an abdominal ultrasound.

The aim of diagnostic peritoneal lavage or abdominal ultrasound is to assist in the decision regarding laparotomy, not the application of an external fixator. In most institutions an external fixator is usually applied in the setting of an operating theatre, whereas diagnostic peritoneal lavage or an abdominal ultrasound can be carried out when the patient presents to the emergency department during the initial assessment and resuscitation. It seems preferable to the authors to do this, and therefore a decision regarding the need for a laparotomy is made at an early stage before the patient is taken to the operating theatre. We trust these comments clarify the basis of the algorithm.

J. F. KEATING, MPhil, FRCS Ed

Royal Infirmary of Edinburgh

Edinburgh, UK.

\section{The type of cement and failure of total hip replacements}

Sir,

In the article by Espehaug et $\mathrm{al}^{1}$ in the August 2002 issue entitled 'The type of cement and failure of total hip replacements', it is stated that CMW gentamicin bone cement contains $0.5 \mathrm{~g}$ of gentamicin per $40 \mathrm{~g}$ pack of polymethylmethacrylate. The correct figure is that each $40 \mathrm{~g}$ pack contains $1 \mathrm{~g}$ (active) of gentamicin. The gentamicin content of CMW cements has been formulated to provide efficacious amounts of gentamicin close to the joint replacement with release characteristics over time to provide optimal prophylaxis against infection. It would have been interesting if the relative rates of infection between the cements with and without gentamicin had been compared in the article. Perhaps this could be addressed in a subsequent follow-up study.

There are some interesting findings concerning the performance of different cements on the outcome of cemented total hip replacements in Norway. While the analyses used included adjustments for potentially important variables, such as the age and gender of the patient and the operative approach, no account was taken of the surgeons' personal cementing technique and cementmixing systems. The importance of the cementing technique has been reported in a number of publications including that of the Swedish Hip Register ${ }^{2}$ in which the application of modern cementing techniques was shown to be associated with improved survivorship. Espehaug et $\mathrm{al}^{1}$ have stated that so-called modern techniques of cementing were commonly used during the period of review. A fundamental aspect of modern cementing techniques concerns the introduction of cement with an appropriately high viscosity. It is of interest that the authors classified Simplex as a high-viscosity bone cement whereas CMW3 was a low-viscosity cement. In fact, when measured on an equivalent time basis, Simplex increases in viscosity at a similar rate to that of CMW3, a rate slower than for Palacos and CMW1 cements. This observation may therefore call into question whether the cementing techniques used for the different types of cement were indeed 'equivalent' and 
'modern' as stated by the authors. If this was the case and modern cementing techniques had not been uniformly adopted, the observations on the performance of individual cements contained in the paper would require significant modification.

N. D. WATKINS, BSc, PhD, MRSC

DePuy CMW

Blackpool, UK.

1. Espehaug B, Furnes O, Havelin LI, Engesaeter LB, Vollset SE. The type of cement and failure of total hip replacements. J Bone Joint Surg [Br] 2002;84-B:832-8.

2. Malchau H, Herberts P. Prognosis of total hip replacement. Presented at the 63rd Annual Meeting of the American Academy of Orthopaedic Surgeons. Feb. 22-26, 1996.

\section{Authors' reply:}

Sir,

We thank Dr Watkins for his letter with comments regarding our paper on the type of cement and failure of total hip replacements. An important issue in our study was the poor results of the CMW cements.

Dr Watkins refers to publications by the Swedish Hip Register concerning the importance of improved cementing techniques regarding the survival of total hip replacement. We acknowledge that if not uniformly adopted, cementing techniques and cement mixing systems are factors which may act as confounders of the results. However, as was stated in the paper, analyses restricted to recent years (1995 to 2000) when modern techniques were in common use, showed similar results to those of earlier years. The Swedish Hip Register has also reported differences in revision probabilities according to the type of cement, with the lowest probability of revision for implants fixed with Palacos cement. ${ }^{1,2}$ Whether the results in our article were due to mechanical problems or handling problems related to the cement, cannot be answered from our study. It is, however, a problem both for patients and surgeons if the instructions from the manufacturer are unclear as to how the cement should be used and may thus lead to unsatisfactory results.

We are aware that in some comparative studies, Simplex cement has been classified as a cement of medium viscosity. ${ }^{3}$ However, because of the previous practice of classifying types of cement as high- or low-viscosity, and since the cement was not marketed as a low-viscosity cement by the manufacturer, we classified the Simplex cement as being of high viscosity.

Our paper states that CMW1 with antibiotic contained $0.5 \mathrm{~g}$ of gentamicin per $40 \mathrm{~g}$ pack. As pointed out by Dr Watkins, the correct figure should be $0.5 \mathrm{~g}$ per $20 \mathrm{~g}$ pack. Although we regret this inaccuracy, it has no bearing on the results of the paper, and we would like to point out that similar results were observed for the CMW1 cement both with and without added gentamicin.

The differences in survival of prostheses presented in the article were based on analyses with all revisions and revisions due to aseptic loosening as the end point. Dr Watkins enquires about results between cements with and without gentamicin if revisions due to infection were chosen as the end point. Our paper did not focus on the effect of antibiotic prophylaxis and revisions due to infection were therefore not analysed. However, it has been demonstrated that among patients receiving systemic antibiotics, the probability of revision due to infection was lower for hip implants fixed with antibiotic-containing cement than for those fixed with plain cement. ${ }^{4}$

B. ESPEHAUG, $\mathrm{MSc}, \mathrm{PhD}$

O. FURNES, MD, PhD

L. I. HAVELIN, MD, $\mathrm{PhD}$

L. B. ENGESÆTER, MD, PhD

The Norwegian Arthroplasty Register

Bergen, Norway.
S. E. VOLLSET, MD, DrPH

University of Bergen

Bergen, Norway.

1. Herberts P, Malchau H. Long-term registration has improved the quality of hip replacement: a review of the Swedish THR Register comparing 160000 cases. Acta Orthop Scand 2000;71:111-21.

2. Malchau H, Herberts P, Söderman P, Odén A. Prognosis of total hip replacement: update and validation of results from the Swedish National Hip Arthroplasty Registry 1979-1998. Scientific exhibition presented at the 67th annual meeting of the American Academy of Orthopaedic Surgeons, Orlando, USA, 2000

3. Kühn K-D. Bone cements: up-to-date comparison of physical and chemical properties of commercial materials. Berlin: Springer-Verlag, 2000.

4. Espehaug B, Engesaeter LB, Vollset SE, Havelin LI, Langeland N Antibiotic prophylaxis in total hip arthroplasty: review of 10905 primary cemented total hip replacements reported to the Norwegian Arthroplasty Register, 1987 to 1995. J Bone Joint Surg [Br] 1997;79-B:590-5.

\section{Intraneural mucoid pseudocysts: a report of ten cases}

Sir,

We read with interest in the September 2001 issue the article by Chick et al ${ }^{1}$ entitled 'Intraneural mucoid pseudocysts: a report of ten cases'. We agree with the authors when they emphasise the necessity of an early diagnosis for a successful surgical outcome. We do not agree, however, with their discussion about the pathogenesis of intraneural cysts and the statement that these cysts do not communicate with an adjacent joint "because such a connection is rarely found and has never been demonstrated by arthro-graphy".

In a published series of 20 ganglion cysts around the knee, we demonstrated the articular origin of a cyst of the tibial nerve at arthrography by performing delayed radiographs and CT. ${ }^{2}$ At the end of routine arthrographic procedures, most of the cysts in our series were not obviously opacified, but diffusion of contrast medium into the cysts was evident on delayed radiographs in 50\% of cases and all cysts were clearly seen to be opacified on CT performed one to two hours after injection of contrast. Delayed CT arthrography showed articular communication of the cysts better than MRI. Indeed, when MRI was available, a communication between the cysts and the joints was obvious in only $25 \%$ of cases. This difficulty of demonstrating a communication between ganglion cysts and joints occurs because the communicating pedicle is narrow.

We recently observed another case of intraneural mucoid cyst (surgically proven), located in the common peroneal nerve. Articular communication was obvious on delayed CT arthrography but not on MRI.

Our findings favour the pathogenic hypothesis of an articular origin of these mucoid cysts. Similar hypotheses have been proposed regarding the pathogenesis of meniscal cysts, which were previously considered as secondary to focal myxoid degeneration, ${ }^{3}$ but subsequently shown to be caused by the migration of synovial fluid through a thin horizontal tear within the meniscus. ${ }^{4}$

\section{J. MALGHEM, MD}

F. E. LECOUVET, MD

B. C. VANDE BERG, MD

C. H. LEBON, MD

B. E. MALDAGUE, MD

Clinique Universitaires St Luc

Brussels, Belgium.

1. Chick G, Alnot J-Y, Silbermann-Hoffman O. Intraneural mucoid pseudocysts: a report of ten cases. J Bone Joint Surg [Br] 2001; 83-B:1020-2.

2. Malghem J, Vande Berg BC, Lebon CH, Lecouvet FE, Maldague BE. Ganglion cysts of the knee: articular communication revealed by delayed radiography and CT after arthrography. Am J Roentgenol 1998;170:1579-83 
3. Lichtenstein L. Diseases of bone and joints. Edited by Mosby Company. 1970;151-4.

4. Barrie HJ. The pathogenesis and significance of meniscal cysts. J Bone Joint Surg [Br] 1979;61-B:184-9.

\section{Author's reply:}

Sir,

We note with interest the comments of Dr Malghem and his colleagues and thank them for their interest. They have demonstrated the articular origin in a case of a cyst of the tibial nerve at arthro- graphy by performing delayed radiographs and CT, and in another case which was surgically proven.

As far as we know, this is the first description of the cause of the problem. The difficulty of demonstrating a communication with the nearby joint is related to the narrow communicating pedicle which can be shown only by delayed radiographs and CT. It also contradicts our statement that there was no evidence of an epithelial lining. We conclude that a communication between the pseudocyst and the joint is rare but must nevertheless be sought.

G. CHICK

Hôpital Bichat

Paris, France.

\section{Fellowship Exam of European Board of Orthopaedics and Traumatology}

The third Fellowship exam of the European Board of Orthopaedics and Traumatology is going to take place in the Onze Lieve Vrouwe Gasthuis in Amsterdam, Holland on the

11 th and 12th October, 2003.

\section{Requirements}

1. Citizenship of European Union (EU) including Norway and Switzerland, and New Countries joining the European Union (EU).

2. Certificate of full qualification as an Orthopaedic Surgeon by the National Authorities (EU Orthopaedic Society).

3. Be fluent in English.

Title

"Fellow of the European Board of Orthopaedics and Traumatology"

\section{Enquiries}

ebotexam@mail.telepac.pt www.ebotexam.20m.com

*Please read the information that will be published in the European Orthopaedic literature or contact your national UEMS-European Board of Orthopaedics and Traumatology delegate.

\section{Application}

Deadline for application will be the 31st of July 2003.

Number of candidates restricted to 40

APPLY NOW!! 